\title{
Hippocampal CA1 pyramidal cells do not receive monosynaptic input from thalamic nucleus reuniens
}

\author{
Lilya Andrianova ${ }^{1,2}$, Erica S Brady ${ }^{1}$, Gabriella Margetts-Smith ${ }^{1}$, Shivali Kohli ${ }^{1}$, Chris J \\ McBain $^{3}$ and Michael T Craig ${ }^{1,2,3}$
}

1. Institute of Biomedical and Clinical Science, University of Exeter Medical School, Prince of Wales Road, Exeter, EX4 4PS, UK

2. Institute of Neuroscience and Psychology, College of Medical, Veterinary and Life Sciences, University of Glasgow, Glasgow G12 8QQ, UK.

3. Program in Developmental Neurobiology, Eunice Kennedy Shriver National Institute of Child Health and Human Development, National Institutes of Health, Bethesda, Maryland 20892, USA

Correspondence to Michael T Craig, mick.craig@glasgow.ac.uk 


\begin{abstract}
Midline thalamic nuclei play a critical role in cognitive functions such as memory, decisionmaking and spatial navigation, by facilitating communication between the many brain regions involved in these processes. One canonical feature of thalamic interactions with the cortex or hippocampus appears to be that the thalamus receives input from, and projects to, excitatory neurons. Thalamic nucleus reuniens (NRe) is located on the midline and is viewed primarily as a relay from prefrontal cortex to hippocampal and entorhinal areas, although these connections are poorly defined at the cellular and synaptic level. Using electrophysiology and monosynaptic circuit-tracing, we found that pyramidal cells in CA1 receive no direct input from NRe. This contrasts starkly with prefrontal cortex, subiculum and entorhinal cortex, and indicates that NRe inputs to CA1 primarily drive local inhibition and not excitation they do in the other regions. The NRe to CA1 projection is thus a unique thalamic projection and as such is raising important questions about the function of NRemediated prefrontal control of the hippocampus.
\end{abstract}

\title{
Introduction
}

Our understanding of the thalamus has evolved far beyond viewing it as a simple relay to acknowledging the important role that it plays in sensory and cognitive functions ${ }^{1}$. For spatial navigation, memory and decision-making, the anterior thalamic nuclei (ATN) and thalamic nucleus reuniens (NRe) play critical, yet complementary, roles $^{2}$. NRe is a midline thalamic nucleus that forms reciprocal connections with medial prefrontal cortex (mPFC) and subiculum, while also projecting to hippocampal region CA1 and entorhinal cortex $(E C)^{2,3}$, thereby providing the principal subcortical relay through which mPFC communicates with the hippocampus proper. Although electrical stimulation in NRe fails to elicit spiking in the CA1 pyramidal layer in vivo ${ }^{4}$, it is widely assumed that NRe targets CA1 pyramidal cells ${ }^{3}$. This assumption is reasonable as we are unaware of any cortical region in which thalamic inputs do not target pyramidal cells. Surprisingly, despite NRe's important role in goal-directed 
spatial navigation ${ }^{5}$ and working memory ${ }^{6}$, NRe projections to CA1 remain poorly defined. We previously reported that both NRe and entorhinal fibres terminate in stratum lacunosummoleculare (SL-M) of CA1 where they target neurogliaform cells ${ }^{7}$. Optogenetic stimulation of $\mathrm{NRe}$ inputs to these neurogliaform cells elicits monosynaptic EPSCs that are defined by large NMDA receptor-mediated components. ${ }^{6}$ We hypothesised that a similarly large NMDA$\mathrm{R}$ component in pyramidal cells could underlie greatly enhanced NRe-fEPSPs in CA1 during EC input coactivation ${ }^{8}$.

\section{Results}

Remarkably, optogenetic activation of NRe axons in CA1 (representative images of axons in supplementary figure 1) using the light-sensitive tools Chronos or Chrimson ${ }^{9}$ (supplementary figure 2) failed to elicit post-synaptic EPSCs in CA1 pyramidal cells (figure 1A \& F), in either ventral CA1 (vCA1; $n=24$, supplementary figure 3$)$ or dorsal $C A 1(d C A 1 ; n=7$, supplementary figure 3). In each slice tested, we only counted an input as negative when we could evoke a post-synaptic response in neurogliaform cells that, with their high NRe input probability, acted as positive controls (figure 1B \& F - H \& supplementary figure 4). In contrast, optogenetic stimulation NRe projections to its other main postsynaptic targets consistently evoked EPSCs in glutamatergic neurons, with mEC principal cells and PFC pyramidal cells displaying significantly larger AMPA receptor-mediated EPSCs than subiculum pyramidal cells or CA1 NGFs (figure $1 \mathrm{C}-\mathrm{H}$ ). Unlike CA1, pyramidal cells in prosubiculum also displayed small NRe-EPSCs in response to optogenetic stimulation, confirming a direct NRe input (supplementary figure 4). We saw no difference in NRe-EPSC amplitude in PFC when comparing NRe input between medial orbital, infralimbic, prelimbic, and anterior cingulate areas (supplementary figure 5). The magnitude of the NRe-EPSC would suggest that the primary target of NRe is prefrontal and entorhinal cortices and not the hippocampus proper, nor possibly even subiculum. 


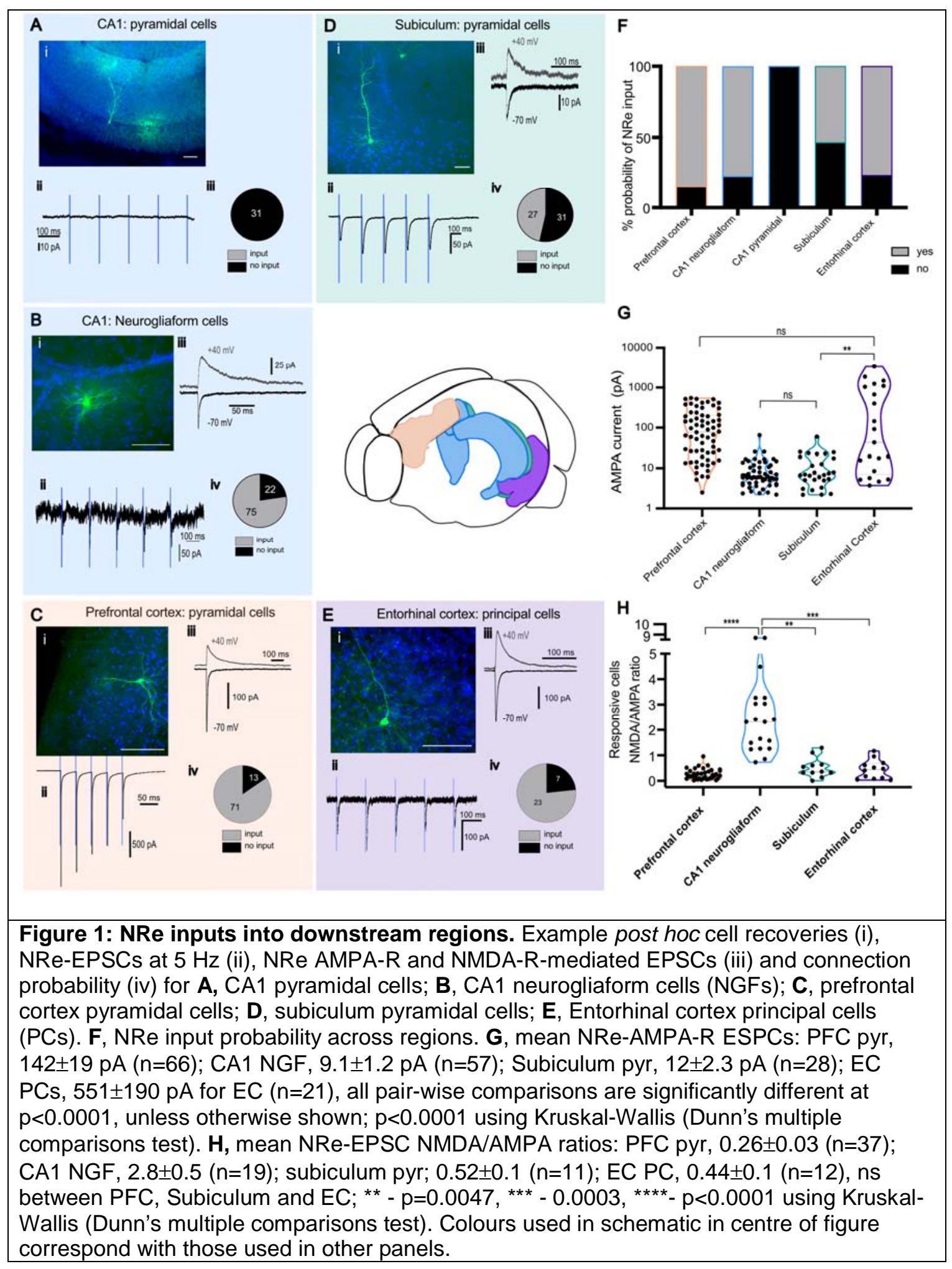




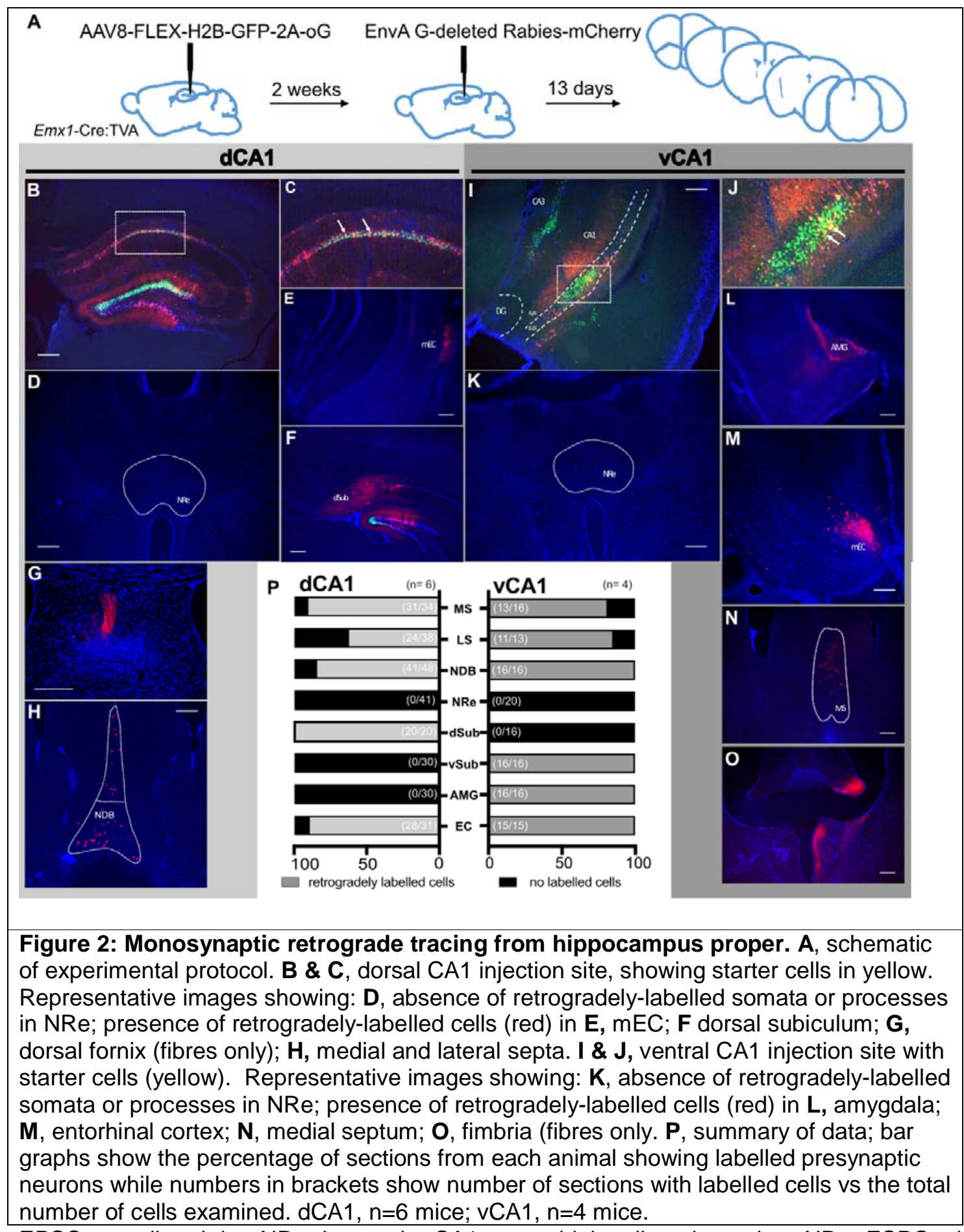

EPSCs mediated by NRe inputs in CA1 pyramidal cells, given that NRe ESPCs in

neurogliaform cells and subiculum pyramidal cells have a small magnitude (figure 1G) we 
could not exclude the possibility that inputs were present but undetectable due to dendritic filtering of the NRe inputs arriving in the distal region of apical dendrites, despite having found no evidence of silent NMDA-R only synapses. Consequently, we carried out rabiesassisted monosynaptic circuit tracing in Emx1-cre mice that conditionally expressed the avian TVA receptor only in pyramidal cells (see methods \& figure 2). We failed to find evidence of monosynaptic NRe inputs to pyramidal cells in either dorsal (figure 2 B, C \& P; $\mathrm{n}=6$ mice) or ventral (figure $2 \mathrm{I}, \mathrm{J} \& \mathrm{P}, \mathrm{n}=4$ ) CA1 but, as expected, we saw consistent retrograde labelling in entorhinal cortices (figure $2 \mathrm{E}, \mathrm{M} \& \mathrm{P}$ ), medial and lateral septa (figure $2 \mathrm{H}, \mathrm{N} \& \mathrm{P}$ ) and subiculum (figure $2 \mathrm{~F}$ \& $\mathrm{P}$; after Sun and colleagues ${ }^{10}$ ). We only observed retrograde labelling of $\mathrm{NRe}$ neurons when injections into ventral hippocampal formation included starter cells in both vCA1 and ventral prosubiculum / subiculum (supplementary figure $6 \mathrm{E}$ to I; $\mathrm{n}=3$; all mice had retrogradely-labelled cells in NRe).

\section{Discussion}

Our findings indicate that NRe does not form monosynaptic projections to pyramidal cells in the $\mathrm{CA} 1$, contrasting greatly with the rest of the prefrontal-hippocampal-entorhinal circuit. This unexpected result contradicts widely-held assumptions about interactions between NRe and hippocampus ${ }^{3,11}$ and is inconsistent with a recent study that used ultrastructural data to show that midline thalamic inputs to CA1 formed 'simple' (and presumably weak) synapses on distal dendrites of dCA1 pyramidal cells ${ }^{12}$. We cannot conclusively state the reason for this discrepancy, although the lack of input to pyramidal cells in our study was very specific to CA1; pyramidal cells in prosubiculum, using Lorente de Nó anatomical definitions ${ }^{13}$, did receive direct input from $\mathrm{NRe}$ (supplementary figure 3). While some regard prosubiculum as the distal region of $\mathrm{CA} 1$, recent transcriptomic studies confirm that it is distinct from both CA1 and subiculum ${ }^{14}$, and our findings support this. There is one report in the literature of midline thalamic stimulation inducing population spikes in $\mathrm{CA} 1^{15}$, but this could be due to feed-forward excitation from mEC. Our data strongly support the assertion that CA1 
pyramidal cells are not the principal target of NRe projections, and that the mechanism through which mPFC exerts control over CA1 is by activating inhibitory interneurons.

So, what is the function of this projection? Prefrontal cortex projections to the hippocampus are important for impulse control ${ }^{16}$, and lesions of $\mathrm{NRe}$ inhibit this function ${ }^{17}$. A recent suggestion for the function of the prefrontal - reuniens - hippocampal circuit is that it actively suppress ongoing memory retrieval ${ }^{18}$, and our study provides a mechanism through which this could occur. Indeed, the large NMDA:AMPA ratio of NRe-EPSCs in neurogliaform cells that we reported here, and previously ${ }^{7}$, suggest that the inhibitory influence evoked via NRe activation is much more likely to be effective in the context of ongoing network activity. Recent behavioural data suggest the requirement for NRe in spatial memory retrieval or "online" spatial processing, but not for off-line consolidation or long-term storage ${ }^{19}$. Given that the largest NRe-mediated EPSCs were present in entorhinal and prefrontal cortices, it could be that NRe suppresses hippocampal activation to facilitate prefrontal - entorhinal communication during associative memory consolidation ${ }^{20}$. Given the large NMDA receptormediated component of NRe-EPSCs onto neurogliaform cells, one is tempted to suggest that the role of NRe could be to increase $\mathrm{Ca}^{2+}$ in NGF dendrites to activate a NO-dependent suppression of inhibition ${ }^{21}$ that could serve to enhance entorhinal-to-CA1 communication. Future behavioural experiments are required to test the function of NRe to CA1 projections, perhaps by using retrograde viruses to allow specific targeting only of those NRe neurons that project directly to CA1.

In summary, we have reported that, unique to all hippocampal or cortical regions, pyramidal cells in hippocampal region CA1 do not receive monosynaptic input from the thalamus. This surprise finding raises important questions for the function of NRe in mediating prefrontal control of the hippocampus.

\section{Acknowledgements}


This work was supported by Biotechnology and Biological Sciences Research Council grant BB/P001475/1 (MTC) and an NIH intramural award (CJM). Salary support from SK was provided by Alzheimer's Research UK Interdisciplinary research grant ARUK-IRG2017B-4 (MTC). GMS and ESB are both GW4 BioMed doctoral training program students funded by the Medical Research Council (MR/N0137941/1). We are grateful to Prof John Aggleton (University of Cardiff) for advice and discussions on anatomy.

\section{Materials and Methods}

Animals: All experiments were conducted in accordance with animal protocols approved by the National Institutes of Health, or in accordance with the UK Animals (Scientific Procedures) Act 1986 after local ethical review by the Animal Welfare and Ethical Review Board at the University of Exeter. We used $N k x 2-1-c r e^{22}$ :RCE or Nkx2-1-cre:Ai9 and Htr3aGFP $^{23}$ mice to target interneurons of MGE or CGE origin, respectively, in electrophysiological experiments. Nkx2-1-cre mice were obtained from Jackson laboratories (C57BL/6J-Tg(Nkx2-1-cre)2Sand/J, stock number 008661) and Htr3a-GFP mice (Tg(Htr3aEGFP)DH30Gsat) were cryo-recovered from MMRRC (NC, USA) and back-crossed onto C57BL/6J mice (Charles River, UK). We used Emx1-cre mice ${ }^{24}$ crossed with floxed TVA mice $^{25}$ to allow specific targeting of pyramidal cells for monosynaptic rabies tracing. Emx1cre mice were obtained from Jackson laboratories (B6.129S2-Emx1 ${ }^{\mathrm{tm} 1(\mathrm{cre}) \mathrm{Kr} \text {, }}$, stock number 005628) and floxed TVA mice (LSL-R26 ${ }^{\text {Tva-lacz }}$ ) were kindly provided by Prof Dieter Saur (Technical University of Munich, Germany). All animals were maintained on a $12 \mathrm{~h}$ constant light / dark cycle and had access to food and water ad libitum and were grouped housed wherever possible. We used standard enrichment that included cardboard tubes, wooden chew blocks and nesting material.

Drugs and chemicals: CGP55845, DNQX, DL-AP5 and picrotoxin were purchased from Tocris Bioscience, and all other chemicals were purchased from Sigma-Aldrich unless otherwise stated. 
Stereotaxic injections for electrophysiology experiments: For optogenetic experiments, we used Nkx2-1-cre:Ai9, Nkx2-1-cre:RCE or Htr3a-GFP mice of both sexes, totalling at 65 mice. with the age at the time of stereotaxic injection ranging from $2-7$ months. Mice of both sexes were used for stereotaxic surgery. Mean weight of the mouse prior to stereotaxic surgery was $26 \mathrm{~g}$ (ranging from $17.5 \mathrm{~g}$ to $49.5 \mathrm{~g}$ ). Two different viruses were used for stereotaxic surgeries: AAV8-hSyn-Chrimson-TdTom (UNC Viral Vector Core, USA, contributed by Ed Boyden; titre $6.3 \times 10^{12}$ viral particles / ml). AAV8-hSyn-Chronos-GFP (UNC Viral Vector Core, USA. contributed by Ed Boyden; titre $3.1 \times 10^{13}$ viral particles $/ \mathrm{ml}$ ).

For the surgery, the mice were anaesthetised with $5 \%$ isoflurane and anaesthesia was maintained with use of 1.5 to $2.5 \%$ isoflurane (flow rate of $\sim 2 \mathrm{Lmin}^{-1} \mathrm{O}_{2}$ ). The mice were placed on a heated pad $(37 \mathrm{C})$ for the duration of the surgery and given $0.1 \mathrm{mg} / \mathrm{kg}$ of buprenorphine (buprenorphine hydrochloride, Henry Schein) subcutaneously at the start of surgery as an adjunct analgesic, plus carprofen $1 \mathrm{mg} / \mathrm{kg}$ (Rimadyl, Henry Schein) was given at a dose of $5 \mathrm{mg} / \mathrm{kg}$ subcutaneously post-surgery and on subsequent days, as required. To target nucleus reuniens, we used the following coordinates: $\mathrm{A} / \mathrm{P}-0.8 \mathrm{~mm}, \mathrm{M} / \mathrm{L} 0.0 \mathrm{~mm}, \mathrm{D} / \mathrm{V}$ $3.8 \mathrm{~mm}$ from pia, with $300 \mathrm{nl}$ of virus (infused at $100 \mathrm{nl} \mathrm{min}{ }^{-1}$ ). After the surgery, the mice were allowed at least a 3-week recovery period to allow sufficient time for the expression of the viral construct. For whole cell patch clamping experiments AAV8-hSyn-Chronos-GFP or AAV8-hSyn-Chrimson-TdTom were used for Nkx2.1-cre:Ai9 or Htr3a-GFP mice, respectively, although a small number of Htr3a-GFP mice received AAV8-hSyn-ChronosGFP to allow direct comparison of EPSC amplitude in the same population of neurons.

Monosynaptic retrograde tracing: For anatomical experiments we used the monosynaptic rabies tracing method that has been previously reported by others ${ }^{10}$. Two mouse lines (Emx1-cre and floxed TVA) were crossed together in order to ensure that the modified rabies virus only targets the pyramidal cells, with Emx1-cre mice used as controls to ensure 
the rabies virus did not transduce neurons in the absence of TVA. A total of 22 mice of both sexes were used, with 2 mice being excluded from the analysis due to failed injections. The age of the mice used ranged from 2 to 6 months, with pre-surgical weights from $18.9 \mathrm{~g}$ to $40.6 \mathrm{~g}$ (mean age 3.5 months, mean weight $24.6 \mathrm{~g}$ ). To highlight the efferent projections from nucleus reuniens to hippocampus, we elected to inject into dorsal and ventral CA1 using the following coordinates: dCA1 was targeted at A/P $-2 \mathrm{~mm}$ (relative to Bregma), M/L $1.5 \mathrm{~mm}$ and $\mathrm{D} / \mathrm{V}-1.35 \mathrm{~mm}$ (from pia) and vCA1 at A/P -2.8 mm (relative to Bregma), M/L -2.4 $\mathrm{mm}$ and D/V-4.2 mm (from pia). The stereotaxic injections of AAV8-FLEX-H2B-GFP-2A-oG (Provided by John Naughton at Salk Institute Viral Vector Core, USA, titre $3.93 \times 10^{12}$ viral particles / ml), followed by injection EnvA G-deleted Rabies-mCherry (Provided by John Naughton at Salk Institute Viral Vector Core, titre $6.13 \times 10^{8}$ viral particles / ml; or from Viral Vector Core facility of the Kavli Institute for Systems Neuroscience, NTNU, Norway, titre 2.6 $\times 10^{10}$ viral particles $/ \mathrm{ml}$ ) 2 weeks after the initial viral injection were performed in the right hemisphere only. The mice were maintained for 2 weeks to provide optimal time for expression, and were killed by transcardial perfusion / fixation with $4 \%$ paraformaldehyde (Cat number P6148 Sigma-Aldrich, UK) in 0.1 M phosphate buffer.

Following the transcardial perfusion, the brains were dissected out and post-fixed for $24 \mathrm{~h}$ in $4 \%$ pfa solution, after which they were cryoprotected using the $30 \%$ sucrose in PBS solution. Once cryoprotected, the brains were sliced at 50 microns using the freezing microtome (Leica, SM2010 R). Selected slices (1 in 5 serially, increasing to 1 in 3 between -0.5 and -1.8 Bregma to ensure thorough representation of nucleus Reuniens of the thalamus across the anterior-posterior axes) were mounted using the Hard Set mounting medium with DAPI (Vectashield, Vector Lab, $\mathrm{H}-1500-10$ ) and the fluorescent fibres were visualised with CoolLED on Nikkon 800 microscope. Representative photos of projections patterns can be found on Supplementary Figure 2. 
Slice preparation and electrophysiology: A minimum of 3 weeks recovery period following the stereotaxic surgery was allowed. Mice were anesthetised with isoflurane and the brain was rapidly dissected out in room temperature NMDG cutting solution, containing (in $\mathrm{mM}$ ): $135 \mathrm{NMDG}, 20$ Choline bicarbonate, 10 glucose, $1.5 \mathrm{MgCl}_{2}, 1.2 \mathrm{KH}_{2} \mathrm{PO}_{4}, 1 \mathrm{KCl}, 0.5 \mathrm{CaCl}_{2}$, saturated with $95 \% \mathrm{O}_{2}$ and $5 \% \mathrm{CO}_{2}(\mathrm{pH}$ 7.3-7.4). Coronal or horizontal slices (400 um) were cut to target prefrontal cortex and dorsal CA1 vs ventral CA1, subiculum and entorhinal cortex, respectively, using a VT-1200S vibratome (Leica Microsystems, Germany). Afterwards, the slices were transferred into a chamber containing recording aCSF, composed of (in mM): $130 \mathrm{NaCl}, 24 \mathrm{NaHCO}_{3}, 3.5 \mathrm{KCl}, 1.25 \mathrm{NaH}_{2} \mathrm{PO}_{4}, 2.5 \mathrm{CaCl}_{2}, 1.5 \mathrm{MgCl}_{2}$, and 10 glucose, saturated with $95 \% \mathrm{O}_{2}$ and $5 \% \mathrm{CO}_{2}(\mathrm{pH} 7.4)$ and placed in a water bath at $37^{\circ} \mathrm{C}$ for 30 minutes, following which they were kept at room temperature until recording.

For recordings individual slices were attached onto $0.1 \%$ poly-L-lysine (Sigma Aldrich, P8920) coated glass slides and placed into the upright microscope and visualized using infrared differential interference contrast microscopy (Olympus BX51 or Scientifica SliceScope). CoolLED pE-4000 system was used to visualise the fibres as well as interneurons, and to provide optogenetic stimulation. The slices were submerged in recording aCSF, warmed to $32-34^{\circ} \mathrm{C}$, and the rate of perfusion was kept at $5 \mathrm{ml} / \mathrm{min}$. The recording electrodes were typically 3-5 $\mathrm{M} \Omega$ size and were pulled from borosilicate glass (World Precision Instruments). The intracellular solution used had the following composition (in mM): 135 Cs-methanesulfonate, $8 \mathrm{NaCl}, 10$ HEPES, 0.5 EGTA, 4 MgATP, 0.3 Na-GTP, $5 \mathrm{QX} 314$, plus $2 \mathrm{mg} / \mathrm{ml}$ biocytin (VWR International, UK), at $\mathrm{pH} 7.25$ adjusted with $\mathrm{CsOH}$ and $285 \mathrm{mOsm}$.

A train stimulation with 5 pulses of $470 \mathrm{~nm}$ or $660 \mathrm{~nm}$ was used to excite the Chronos ${ }^{26}$ or Chrimson opsins, respectively. The presence or absence of responses was recorded in voltage clamp mode. Cells that were found to have a response to a train stimulation were then switched onto repeated single pulse protocol (ISI of $10 \mathrm{~s}$ ), and the AMPA response was 
recorded at a holding potential of $-70 \mathrm{mV}$. GABA-R antagonists were bath applied from the start in hippocampus and subiculum, but not in EC or PFC due to epileptiform activity being observed upon NRe stimulation with GABA-R antagonists present. The extracellular $\mathrm{GABA}_{\mathrm{A}}$ and $\mathrm{GABA}_{B}$ receptor antagonists used were picrotoxin $(100 \mu \mathrm{M})$ and CGP55845 $(1 \mu \mathrm{M}) .10$ $\mu \mathrm{m}$ of DNQX was added to abolish the AMPA current at $-70 \mathrm{mV}$, after which the cell was switched to $+40 \mathrm{mV}$ to record the NMDA current. To confirm the identity of NMDA current, DAP $5(100 \mu \mathrm{M})$ was added at the end of the recording. Whole-cell patch-clamp recordings were made using a Multiclamp 700A or 700B amplifier (Molecular Devices, Sunnyvale, CA). Signals were filtered at $3 \mathrm{kHz}$ and digitized at $10 \mathrm{kHz}$ using a Digidata 1322A or $1440 \mathrm{~A}$ and pClamp 9.2 or 10.2 (Molecular Devices, USA). Recordings were not corrected for a liquid junction potential. The recordings were then imported into IgorPro (Wavemetrics, OR) using Neuromatic (Thinkrandom, UK) for further analysis.

Data Analysis: For quality control, cells with changes in input resistance of over $20 \%$ were excluded from the data analysis. The AMPA receptor-mediated EPSC was determined as the maximal EPSC peak at $-70 \mathrm{mV}$ and NMDA receptor-mediated EPSC as the highest peak at $+40 \mathrm{mV}$. GraphPad Prism (Graphpad, CA) was used for statistical analysis. Data were tested for normality using the D'Agostino and Pearson test and subsequently analysed by parametric or nonparametric tests as appropriate. Unless otherwise stated, all values are mean \pm SEM.

\section{References}

1. Sherman, S. M. The thalamus is more than just a relay. Curr Opin Neurobiol 17, 417-422 (2007).

2. Mathiasen, M. L., O'Mara, S. M. \& Aggleton, J. P. The anterior thalamic nuclei and nucleus reuniens: So similar but so different. Neurosci Biobehav Rev 119, 268-280 (2020).

3. Weel, M. J. D. der et al. The nucleus reuniens of the thalamus sits at the nexus of a hippocampus and medial prefrontal cortex circuit enabling memory and behavior. Learn Memory 26, 191-205 (2019). 
4. Weel, M. J. D.-V. der, Silva, F. H. L. da \& Witter, M. P. Nucleus Reuniens Thalami Modulates Activity in Hippocampal Field CA1 through Excitatory and Inhibitory Mechanisms. J Neurosci 17, 5640-5650 (1997).

5. Ito, H. T., Zhang, S.-J., Witter, M. P., Moser, E. I. \& Moser, M.-B. A prefrontalthalamo-hippocampal circuit for goal-directed spatial navigation. Nature 522, 50-55 (2015).

6. Duan, A. R. et al. Delta Frequency Optogenetic Stimulation of the Thalamic Nucleus Reuniens Is Sufficient to Produce Working Memory Deficits: Relevance to Schizophrenia. Biol Psychiat 77, 1098-1107 (2015).

7. Chittajallu, R. et al. Afferent specific role of NMDA receptors for the circuit integration of hippocampal neurogliaform cells. Nat Commun 8, 152 (2017).

8. Weel, M. J. D. der, Silva, F. H. L. da \& Witter, M. P. Interaction of nucleus reuniens and entorhinal cortex projections in hippocampal field CA1 of the rat. Brain Struct Funct 222, 2421-2438 (2017).

9. Klapoetke, N. C. et al. Independent optical excitation of distinct neural populations. Nat Methods 11, 338-346 (2014).

10. Sun, Y. et al. Cell-Type-Specific Circuit Connectivity of Hippocampal CA1 Revealed through Cre-Dependent Rabies Tracing. Cell Reports 7, 269-280 (2014).

11. Cassel, J.-C. et al. The reuniens and rhomboid nuclei: Neuroanatomy, electrophysiological characteristics and behavioral implications. Prog Neurobiol 111, 34-52 (2013).

12. Bloss, E. B. et al. Single excitatory axons form clustered synapses onto CA1 pyramidal cell dendrites. Nat Neurosci 21, 353-363 (2018).

13. Lorente de Nó, R. Studies on the Structure of the Cerebral Cortex II. Continuation of the Study of the Ammonic System. J Psychol. Neurol. 46, 113-177 (2002).

14. Ding, S.-L. et al. Distinct Transcriptomic Cell Types and Neural Circuits of the Subiculum and Prosubiculum along the Dorsal-Ventral Axis. Cell Reports 31, 107648 (2020).

15. Bertram, E. H. \& Zhang, D. X. Thalamic excitation of hippocampal CA1 neurons: a comparison with the effects of CA3 stimulation. Neuroscience 92, 15-26 (1999).

16. Chudasama, Y., Doobay, V. M. \& Liu, Y. Hippocampal-Prefrontal Cortical Circuit Mediates Inhibitory Response Control in the Rat. J Neurosci 32, 10915-10924 (2012).

17. Prasad, J. A., Macgregor, E. M. \& Chudasama, Y. Lesions of the thalamic reuniens cause impulsive but not compulsive responses. Brain Struct Funct 218, 8596 (2013). 
18. Anderson, M. C., Bunce, J. G. \& Barbas, H. Prefrontal-hippocampal pathways underlying inhibitory control over memory. Neurobiol Learn Mem 134, 145-161 (2016).

19. Mei, H., Logothetis, N. K. \& Eschenko, O. The activity of thalamic nucleus reuniens is critical for memory retrieval, but not essential for the early phase of "offline" consolidation. Learn Memory 25, 129-137 (2018).

20. Takehara-Nishiuchi, K., Maal-Bared, G. \& Morrissey, M. D. Increased Entorhinal-Prefrontal Theta Synchronization Parallels Decreased EntorhinalHippocampal Theta Synchronization during Learning and Consolidation of Associative Memory. Front Behav Neurosci 5, 90 (2012).

21. Li, G., Stewart, R., Canepari, M. \& Capogna, M. Firing of Hippocampal Neurogliaform Cells Induces Suppression of Synaptic Inhibition. J Neurosci 34, 1280-1292 (2014).

22. Xu, Q., Tam, M. \& Anderson, S. A. Fate mapping Nkx2.1-lineage cells in the mouse telencephalon. J Comp Neurol 506, 16-29 (2008).

23. Gong, S. et al. A gene expression atlas of the central nervous system based on bacterial artificial chromosomes. Nature 425, 917-925 (2003).

24. Guo, H. et al. Specificity and Efficiency of Cre-Mediated Recombination in Emx1-cre Knock-in Mice. Biochem Bioph Res Co 273, 661-665 (2000).

25. Seidler, B. et al. A Cre-loxP-based mouse model for conditional somatic gene expression and knockdown in vivo by using avian retroviral vectors. Proc National Acad Sci 105, 10137-10142 (2008).

26. Klapoetke, N. C. et al. Independent optical excitation of distinct neural populations. Nat Methods 11, 338-346 (2014). 